\title{
Development of an untargeted DNA adductomics method by ultra-high performance liquid chromatography coupled to high-resolution mass spectrometry
}

\author{
Giorgia La Barbera ${ }^{\mathrm{a}}$ and Lars Ove Dragsted*a . \\ e-mail:1dra@nexs.ku.dk \\ ${ }^{a}$ Department of Nutrition Exercise and Sports, University of Copenhagen, Frederiksberg, DK-1985
}

\section{- ABSTRACT}

Cancer may be initiated by covalent modification of DNA by genotoxic molecules coming from diet, environment, inflammation, and other sources. For most of these genotoxicants there is little evidence of their identity. DNA adductomics is a new research field, aiming to screen for unknown DNA adducts by high resolution mass spectrometry (HRMS). However, due to the low abundance of DNA adducts, DNA adductomics presents several analytical challenges. In this work, a sensitive untargeted DNA adductomics method was developed by using ultra-high performance liquid chromatography (UHPLC) coupled via an electrospray ionization source (ESI) to a quadrupole-time of flight MS instrumentation. Mobile phases with ammonium bicarbonate gave the best signal enhancement. The MS capillary voltage, cone voltage and detector voltage had most effect on the response of the DNA adducts. A low adsorption vial was selected for reducing analyte loss. A hybrid surface coated HSST3 premier column was tested for reducing adsorption of the DNA adducts. The optimized method was applied to analyse DNA adducts in calf thymus and cat colon DNA by performing a $\mathrm{MS}^{\mathrm{E}}$ acquisition and screening for loss of deoxyribose, both in-source and in the fragmentation spectra, and for the nucleobase fragment ions. The putative DNA adducts were matched with an inhouse DNA adduct database. Thirteen DNA adducts were observed in DNA from calf thymus and cat colon, four of them never reported before, showing promise for the application of this untargeted method in future

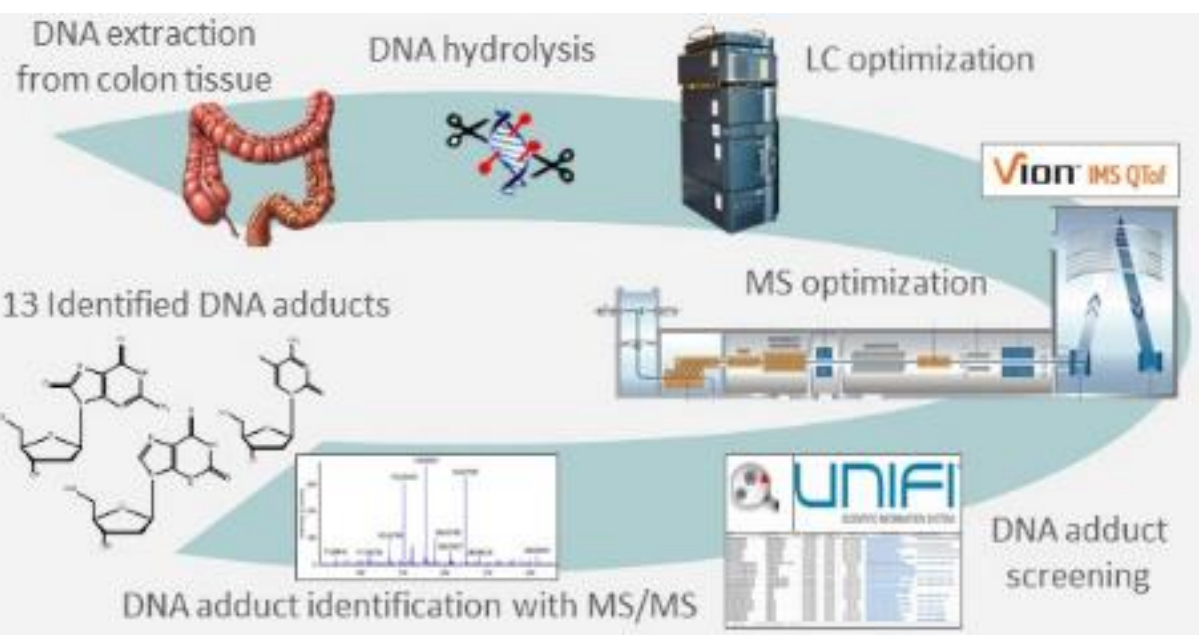
human studies.

\section{- INTRODUCTION}

DNA adductomics, a new -omics science exploring the modifications of DNA from endogenous or exogenous genotoxicants, has developed over the past few years. ${ }^{1}$ The exposure of human DNA to genotoxic chemicals induces the formation of covalent DNA adducts which, if not repaired, can lead to gene mutations, ultimately increasing the risk of cancer. ${ }^{2}$ The measurement of DNA adducts is of fundamental importance in assessing the potential effect of exposure to endogenous carcinogens and exogenous ones from the diet and the environment, and in understanding their mechanisms of action. ${ }^{1}$

Several analytical methods, such as immunochemical methods, ${ }^{32} \mathrm{P}$-postlabeling techniques, and liquid chromatography coupled to mass spectrometry (LCMS), have been used for DNA adduct analysis, and LC-MS is now considered to be the gold standard technique. However, the limitations of older MS instruments in terms of both sensitivity and selectivity allowed monitoring of only a few targeted DNA adducts at a time, not providing a global picture of the 
"DNA adductome". ${ }^{3}$ With modern high resolution mass spectrometry (HRMS), providing high sensitivity and accurate compound mass, DNA adducts can be identified with high confidence. HRMS, together with ultra-high performance (UHP)LC, have opened new horizons in the screening of unknown DNA adducts and led to the development of this new research field. ${ }^{4}$ DNA adductomics by UHPLC-HRMS presents new challenges, demanding new sample preparation protocols, chromatographic methods, data acquisition, and data analysis approaches. The major analytical challenge in DNA adductomics is still the need for high sensitivity and selectivity, as DNA adducts are present at trace levels and in a very complex matrix. ${ }^{3,4}$ Indeed, while selective extraction of the analytes of interest can be performed in targeted methods, this is not possible for untargeted analyses, resulting in severe matrix effects and reduced sensitivity. Another challenge in DNA adductomics is the screening and identification of DNA adducts. Whereas traditional -omics sciences rely on extensive existing database and software support, DNA adductomics is in a developmental phase. ${ }^{5,6} \mathrm{~A}$ few studies have been published with the aim of building DNA adduct databases, which can be used as a tool for DNA adduct profiling..$^{7-9} \mathrm{~A}$ few other studies reported the development of untargeted DNA adductomics methods for identification of unknowns. ${ }^{10-13}$

In this present work, we focus on screening for unknown DNA adducts, primarily to initiate studies related to colorectal cancer (CRC). Previous analyses of colon tissues with older techniques reported DNA adducts coming from red meat intake, alcohol intake, and smoking. ${ }^{14}$ Although the studies showed promising results, the association of CRC with these exposures has only been partially elucidated,,${ }^{15}$ and more advanced methods for profiling the colon epithelial DNA adductome are needed.

We report here a sensitive UHPLC-HRMS based method for this purpose. The chromatographic and mass spectrometric conditions of an UHPLC coupled to a quadrupole-time of flight MS (Vion-qTOF) via an electrospray ionization source (ESI), were optimized by using a mixture of DNA adduct reference standards, in order to increase the sensitivity of the instrumental analysis. In addition, we implemented an $\mathrm{MS}^{\mathrm{E}}$ acquisition for the untargeted screening of DNA adducts. Finally, we applied the developed method for the analysis of DNA adducts in calf thymus and cat colon DNA.

\section{- EXPERIMENTAL SECTION \\ Chemicals and materials}

Milli-Q ultra-pure water (Merck Life Sciences, Søborg, Denmark), methanol optima LC/MS grade from Thermo Fisher Scientific (Waltham, MA), and formic acid, acetic acid, ammonium acetate, ammonium formate, and ammonium bicarbonate from Merck (St. Louis, MO.) were used for the UHPLC analysis.

The following DNA adducts and nucleosides, reference standards, were purchased from Toronto Research Chemicals: 2'-deoxyadenosine monohydrate (dA); 2'-deoxyguanosine monohydrate (dG); thymidine (dT); 2'-deoxyuridine (dU); 2'-deoxy-N6methyladenosine (N6-Me-dA); 5-methyl-2'deoxycytidine (5-Me-dC); O6-methyl-2'deoxyguanosine (O6-Me-dG); 2'-deoxy-N3methyluridine (N3-Me-dU); N3-methylthymidine (N3-Me-dT); N4,5-dimethyldeoxycytidine (N4,5DiMe-dC); N2-ethyl-2'-deoxyguanosine (N2-ethyldG); N6-(2-hydroxyethyl)-2'-deoxyadenosine (N6-2OH-ethyl-dA); 8-oxo-2'deoxyguanosine (8-oxo-dG); etheno-2'-deoxy- $\beta$-D-adenosine (1,N6- $-\mathrm{dA}) ; 3, \mathrm{~N} 4-$ etheno-2'-deoxycytidine (3,N4- $\varepsilon$-dC); 3-(2-deoxy- $\beta$ D-erythro-pentofuranosyl)pyrimido[1,2-a]purin-

10(3H)-one (M1-dG); 3-(2-Deoxy- $\beta$-D-erythropentofuranosyl)-3,5-dihydropyrimido[1,2-a]purine-

6,10-dione (6-Oxo-M1-dG); $\gamma$-Hydroxy-1,N2propano-2'-deoxyguanosine $(\gamma-\mathrm{OH}-1, \mathrm{~N} 2-\mathrm{PdG})(\gamma-\mathrm{OH}-$ Acr-dG); $\quad \alpha$-Methyl- $\gamma$-hydroxy-1,N2-propano-2'deoxyguanosine (mixture of diastereomers) $(\alpha-\mathrm{Me}-\gamma-$ OH-1,N2-PdG) (Cro-dG); N-(2'-deoxyguanosin-8yl)-4-aminobiphenyl (C8-ABP-dG); and N2(deoxyguanosin-8-yl)-2-amino-3,8-

dimethylimidazo[4,5-f] quinoxaline (C8-MeIQx-dG). The following nucleoside reference standards were purchased from Merck: adenosine, cytidine (dC), uridine. Stock solutions of the DNA adduct standards were dissolved at 1 or $0.5 \mathrm{mg} \mathrm{mL}^{-1}$ in methanol, or a mixture of water and methanol. A stock solution was prepared containing all the standards at $20 \mu \mathrm{g} \mathrm{mL}^{-1}$. The working solutions were diluted with water to concentrations ranging from $100 \mathrm{ng} \mathrm{ml}^{-1}$ to $1 \mathrm{ng} \mathrm{ml}^{-1}$. 
The following products for DNA extraction and hydrolysis were purchased from Merck: Ribonuclease A from bovine pancreas for molecular biology; Proteinase K from Tritirachium album BioUltra, for molecular biology; Sodium dodecyl sulfate (SDS) BioUltra, for molecular biology; Phenol:chloroform:isoamyl alcohol mixture (25:24:1, $v / v / v) \quad$ BioUltra, for molecular biology; Deoxyribonucleic acid sodium salt from calf thymus, Type I; Deoxyribonuclease I from bovine pancreas, Type IV (DNaseI); Phosphodiesterase I from Crotalus atrox (Western Diamondback Rattlesnake), Type IV (PDEI); Alkaline Phosphatase from bovine intestinal mucosa (AP); Tris(hydroxymethyl)aminomethane hydrochloride (Tris $\mathrm{HCl}$ ); ethylenediaminetetraacetic acid (EDTA); sodium chloride ( $\mathrm{NaCl}$ ); and magnesium chloride hexahydrate $\mathrm{MgCl}_{2} \quad 6 \mathrm{H}_{2} \mathrm{O}$. Ethanol was purchased from Thermo Fisher Scientific (Waltham, MA).

\section{DNA from calf thymus experiment}

DNA from calf thymus was hydrolyzed before analysis. In brief, $0.5 \mathrm{mg}$ of DNA was dissolved in 1 $\mathrm{mL}$ of incubation buffer $(10 \mathrm{mM}$ Tris- $\mathrm{HCl}$ and $5 \mathrm{mM}$ $\mathrm{MgCl}_{2}$, adjusted at $\mathrm{pH}$ 7). Five hundred units of DNaseI were added and the sample was incubated overnight in a mixer at $37^{\circ} \mathrm{C}$. The next day, an additional 500 units of DNaseI were added to the sample, together with 0.01 units of PDEI and 100 units of AP. The sample was incubated overnight in a mixer at $37^{\circ} \mathrm{C}$. The next day, two volumes of cold methanol were added to the sample to precipitate the proteins. The supernatant was collected and evaporated. The sample was dissolved with a total of $300 \mu \mathrm{L}$ of $\mathrm{H}_{2} \mathrm{O}: \mathrm{MeOH}(90: 10, v / v)$.

\section{DNA from cat colon}

A colon sample was obtained from a cat that was euthanized at the owners request at the University Hospital for Companion Animals, University of Copenhagen. The owner signed an informed consent allowing that the cat be used for teaching and research purposes following euthanasia. The colon epithelial tissue was scraped off the resected colon and $500 \mathrm{mg}$ were ground in liquid nitrogen. The sample was dissolved in $2.5 \mathrm{~mL}$ of the DNA digestion buffer (50 $\mathrm{mM}$ TRIS HCl, $10 \mathrm{mM}$ EDTA and $100 \mathrm{mM} \mathrm{NaCl}$, adjusted at pH 8). SDS $300 \mu \mathrm{L} 10 \%$, and 250 units of proteinase $\mathrm{K}$ were added to the sample and incubated over night at $37^{\circ} \mathrm{C}$. The next day, 250 units of RNase were added and incubated for two hours at $37^{\circ} \mathrm{C}$. DNA was extracted from the sample with 1 volume of phenol:chloroform:isoamyl alcohol $(25: 24: 1, v / v / v)$ and mixed by inversion. After centrifugation at $1600 \mathrm{~g}$ for $10 \mathrm{~min}$, the upper phase was collected. Two volumes of cold EtOH were added, and the vial was inverted for DNA precipitation. After centrifugation at $1600 \mathrm{~g}$ for $10 \mathrm{~min}$, the supernatant was discarded and the DNA was washed with $3 \mathrm{~mL}$ of $\mathrm{EtOH}: \mathrm{H}_{2} \mathrm{O}$ (70:30, $v / v)$. The sample was inverted again, followed by centrifugation at $1600 \mathrm{~g}$ for $5 \mathrm{~min}$. The supernatant was discarded, and the sample was air dried at room temperature for 10-15 min. The DNA was dissolved in $10 \mathrm{mM}$ Tris- $\mathrm{HCl}, 5 \mathrm{mM} \mathrm{MgCl}_{2}$, adjusted to $\mathrm{pH}$ 7, and left overnight at $4^{\circ} \mathrm{C}$. The following day, the DNA extraction yield $(0.89 \mathrm{mg})$ and purity $\left(\mathrm{OD}_{260 / 280}=1.87\right.$ $\mathrm{OD}_{260 / 230}=2.12$ ) were assessed by UV. The equivalent of $0.5 \mathrm{mg}$ of DNA was used for the subsequent DNA hydrolysis procedure, as described for DNA from calf thymus.

\section{UHPLC-HRMS method optimization}

The analysis was performed on an $\mathrm{H}$ class Acquity UHPLC coupled to a Vion-IMS-qTOF (Waters, Milford, MA) via a heated electrospray ionization (ESI) source. The UHPLC system was equipped with a quaternary pump and an autosampler thermostated at $10^{\circ} \mathrm{C}$. A C18 HSS T3 column $(100 \times 2.1 \mathrm{~mm}, 1.8 \mu \mathrm{m}$ particle size) (Waters) was used at $0.4 \mathrm{~mL} \mathrm{~min}^{-1}$ and at $50^{\circ} \mathrm{C}$.

Different mobile phases were compared in order to improve chromatographic separation and sensitivity, and to decrease in-source fragmentation and adduct formation. Details of the mobile phase comparison experiment are provided in the Supporting Information (Table S1). The best chromatographic condition used $10 \mathrm{mM} \mathrm{NH}_{4} \mathrm{HCO}_{3}$ as mobile phase $\mathrm{A}$, and $\mathrm{MeOH}$ with $10 \mathrm{mM} \mathrm{NH}_{4} \mathrm{HCO}_{3}$ as mobile phase B.

Several MS spectrometric parameters and acquisitions were evaluated to optimize the sensitivity and to decrease the in-source fragmentation of DNA adducts. Different values of capillary voltage, sampling cone voltage, source temperature, desolvation temperature, desolvation gas, collision energy, mass range, profile or automatic mode in the quadrupole isolation, and detector voltage were tested as summarized in the Supporting Information (Table S2). The optimal tuning 
parameters of the Vion-IMS-qTOF were: capillary voltage $0.5 \mathrm{kV}$; sampling cone voltage $20 \mathrm{~V}$; source temperature $110{ }^{\circ} \mathrm{C}$; desolvation temperature $600{ }^{\circ} \mathrm{C}$; desolvation gas $800(\mathrm{~L} / \mathrm{h})$; collision energy $6 \mathrm{eV}$; cone gas $50(\mathrm{~L} / \mathrm{h})$. The detector voltage was set to $3000 \mathrm{~V}$. The Vion-IMS-Q-TOF was operated in $\mathrm{MS}^{\mathrm{E}}$ acquisition mode and samples were acquired in positive polarity mode. For both the low and high energy trace, the scan range was $50-1000 \mathrm{~m} / \mathrm{z}$ and the scan time $0.4 \mathrm{~s}$. For the high energy trace, optimization of the collision energy was performed to obtain an informative fragmentation pattern, finally choosing a mass energy ramp ranging from 20 to $50 \mathrm{eV}$.

Finally, in order to reduce eventual adsorption processes of DNA adducts, low adsorption injection vials and a low adsorption column were tested by analysing the mix of DNA adduct reference standards at concentrations between 1 and $10 \mathrm{ng} \mathrm{ml}^{-1}$. Five different injection vials - LC-MS certified clear glass Vial, TruView Vial and Quan Recovery Max Peak (all Waters), Low Adsorption Vial (Supelco, Merck, St. Louis, MO), and Reduced Surface Activity RSA-Pro Vial (Microsolv, Greater Wilmington, NC) - were compared by performing 14 repeated injections over a period of $24 \mathrm{hrs}$. The Low Adsorption Vial gave the least adsorption and the highest stability of the signal over time, and was chosen for the subsequent column comparison. Two hybrid surface coated Premier C18 HSS T3 columns $(100 \times 2.1 \mathrm{~mm}, 1.8 \mu \mathrm{m}$ particle size $)$ (Waters) were compared with the HSS T3 column used in previous experiments. The latter gave better results and was then used for analysis of real samples.

The final optimized conditions were used for the analysis of the hydrolyzed DNA from calf thymus and from cat colon, using the following gradient: 0-1 min (5\% B), 1-21 $\min (0-99 \%$ B), followed by a $2 \mathrm{~min}$ wash at $99 \% \mathrm{~B}$ and 2 min equilibration at $5 \% \mathrm{~B}$.

The mass spectrometer was periodically cleaned, and externally calibrated every 2 weeks using the calibration solution Major Mix (Waters). Lock mass correction was applied continuously during the runs by injecting $15 \mu \mathrm{L} \quad \mathrm{min}^{-1}$ of $100 \mathrm{ngmL}^{-1}$ leucine/enkephalin (Waters) every 5 minutes. Three technical replicates were performed for each condition evaluated. For the evaluation of the chromatographic conditions, the three replicates were run after flowing the mobile phase for 1 hour and running two blanks for assuring column conditioning. Each different additive was evaluated at increasing concentrations on the same day. To avoid instrumental variability, the best concentration of each additive was chosen and compared with the others on the same day. To minimize column passivation in the evaluation of the mass spectrometric conditions and for vial comparisons, a highly concentrated DNA adduct mix was injected continuously over 4 hours before the runs. To see which column had the least passivation effect, a blank was injected continuously over 4 hours before the comparison. Injection volume for all analyses was $5 \mu \mathrm{L}$.

\section{Data analysis and DNA adduct identification}

Raw data files obtained for the optimization of the chromatographic and mass spectrometric parameters were acquired by UNIFI software (version 1.9.4.053) (Waters), and transformed into .mzml format using MSconvert(http://proteowizard.sourceforge.net/tools.s html). ${ }^{16}$ The converted files were then imported into MZmine (http://mzmine.github.io) for further analysis. ${ }^{17}$ Values such as peak area, full width at half maximum (FWHM), and the asymmetry factor, were extrapolated from the analyzed chromatographic runs. Briefly, the Targeted Peak Detection module was used for integrating the peaks related to the adducts and insource fragments of the DNA adduct standards. Peak integration was checked and manually corrected when necessary. The RANSAC alignment was used for aligning the chromatographic runs acquired under the same chromatographic conditions. The Join Aligner was used for aligning runs acquired with different chromatographic conditions, so retention time could be omitted from the alignment. Parameters used for data analysis in MZmine are reported in the Supporting Information (Table S3.1). Retention time, peak area of every single adduct, and ratio of $[\mathrm{M}+\mathrm{H}]^{+}$over any other adduct or in-source fragment, full width at half maximum (FWHM), asymmetry factor, peak capacity, and resolution, were extrapolated as reported elsewhere, ${ }^{18}$ and used for the comparison. See Supporting Information S3 for details on the calculations.

Raw data files related to the analysis of DNA from calf thymus and cat colon were acquired and analyzed by UNIFI software (version 1.9.4.053) (Waters) in four 
steps. The first step consisted of peak picking and alignment of the chromatographic runs. The second step was a search for possible adducts. These were chosen based on the typical ionization behavior of the DNA adduct reference standards in the low energy trace. $[\mathrm{M}-\mathrm{dR}+\mathrm{H}]^{+}$was included in the adducts, even though it is formally an in-source fragment due to the loss of deoxyribose (-dR). The third step was a search for the neutral loss of - $\mathrm{dR}$ between the low and the high energy trace. The fourth step was a search for common fragments, i.e. the ion masses related to the unmodified nucleobases, adenine, cytosine, guanine, thymine, and uracil in the high energy trace. The final and fifth step was matching of all features with an in-house database that had been built upon a literature research ${ }^{7-9}$ and uploaded into UNIFI beforehand. The parameters for the data analysis with UNIFI are reported in the Supporting Information (Table S3.2). A manual investigation of the fragmentation spectra of the selected compounds was performed by running a targeted MS2 acquisition with a collision energy ramp of 20-50 eV. Identification confidence levels were associated with the DNA adducts based on the work of Schymanski et al. ${ }^{19}$, but with some modifications: level 1) for compounds identified by reference standard retention time and MS2 spectra comparison; level 2) for compounds showing i) the loss of $-\mathrm{dR}$ in both MS1 and MS2, ii) product ions in MS2 deriving from the DNA adduct modification, iii) product ions in MS2 typical of one of the 5 nucleobases; level 3) was assigned to compounds showing the typical fragmentation pattern of DNA adducts, i.e. the loss of $-\mathrm{dR}$ in both MS1 and MS2, but with no informative fragments in MS2 related to the DNA adduct modification and to the nucleobase.

\section{- RESULTS AND DISCUSSION}

Early studies on DNA damage mainly used ${ }^{32} \mathrm{P}$ postlabeling methods to screen for and measure adduct levels. More recently, UHPLC-HRMS has become the gold standard for DNA adduct analysis, especially because it provides reliable identification of DNA adducts and can perform untargeted DNA adductomics ${ }^{4}$. Although this is a promising technique, method development is still ongoing, especially for improving instrumental sensitivity. Measurements of up to 1 DNA adduct in $10^{10}$ unmodified nucleotides have been accomplished by targeted methods ${ }^{20}$, but untargeted methods do not reach similar levels of sensitivity. ${ }^{9}$ To develop a sensitive untargeted method, we therefore optimized several chromatographic and mass spectrometric parameters. The formation of adducts such as $[\mathrm{M}+\mathrm{H}]^{+},[\mathrm{M}+\mathrm{Na}]^{+},[\mathrm{M}+\mathrm{K}]^{+},[2 \mathrm{M}+\mathrm{H}]^{+}$, $[2 \mathrm{M}+\mathrm{Na}]^{+}$, and high levels of the in-source fragment $[\mathrm{M}-\mathrm{dR}+\mathrm{H}]^{+}$, was detected from 25 DNA adducts. The aim of this study was therefore to improve the response of the DNA adducts by increasing $[\mathrm{M}+\mathrm{H}]^{+}$, while simultaneously decreasing the other adducts and the $[\mathrm{M}-\mathrm{dR}+\mathrm{H}]^{+}$fragment.

\section{Optimization of Chromatographic Conditions}

Several mobile phases have been used in the past for the analysis for DNA adducts. These include water $\left(\mathrm{H}_{2} \mathrm{O}\right)$ as mobile phase $\mathrm{A}$, and methanol $(\mathrm{MeOH})$ or acetonitrile (ACN) as mobile phase $\mathrm{B}$, both phases either with or without the addition of acetic acid $\left(\mathrm{CH}_{3} \mathrm{COOH}\right)$, formic acid $(\mathrm{HCOOH})$, or ammonium acetate $\left(\mathrm{CH}_{3} \mathrm{COONH}_{4}\right){ }^{9}{ }^{921-23}$ However, to the best of our knowledge, there is no study showing a comprehensive comparison of the commonly used chromatographic conditions for the analysis of different classes of DNA adducts. Only one study reports the comparison of ammonium acetate, formate $\left(\mathrm{HCOONH}_{4}\right)$ and bicarbonate $\left(\mathrm{NH}_{4} \mathrm{HCO}_{3}\right)$ for the analysis of acetaldehyde DNA adducts, showing the last to be the most promising additive. ${ }^{24}$ This was confirmed in another study, where HILIC chromatography was used for detecting acrolein DNA adducts. ${ }^{25}$ Based on this, we tested chromatographic conditions using different concentrations of $\mathrm{HCOOH}$, $\mathrm{CH}_{3} \mathrm{COOH}, \mathrm{CH}_{3} \mathrm{COONH}_{4}, \mathrm{HCOONH}_{4}$ and $\mathrm{NH}_{4} \mathrm{HCO}_{3}$, in the mobile phases (Table $\mathrm{S} 1$ ). ACN as phase $\mathrm{B}$ was excluded because $\mathrm{MeOH}$ showed much higher ionization efficiency in preliminary experiments. The mobile phase evaluation was carried out only in positive polarity mode, as most of the DNA adducts showed better ionization in positive mode. Each additive was evaluated at different concentrations and then the best concentration of each additive was compared with the others. Although the intensities of $[\mathrm{M}+\mathrm{H}]^{+}$and $[\mathrm{M}-\mathrm{dR}+\mathrm{H}]^{+}$were changed by using different mobile phases, the ratio between $[\mathrm{M}+\mathrm{H}]^{+}$and 


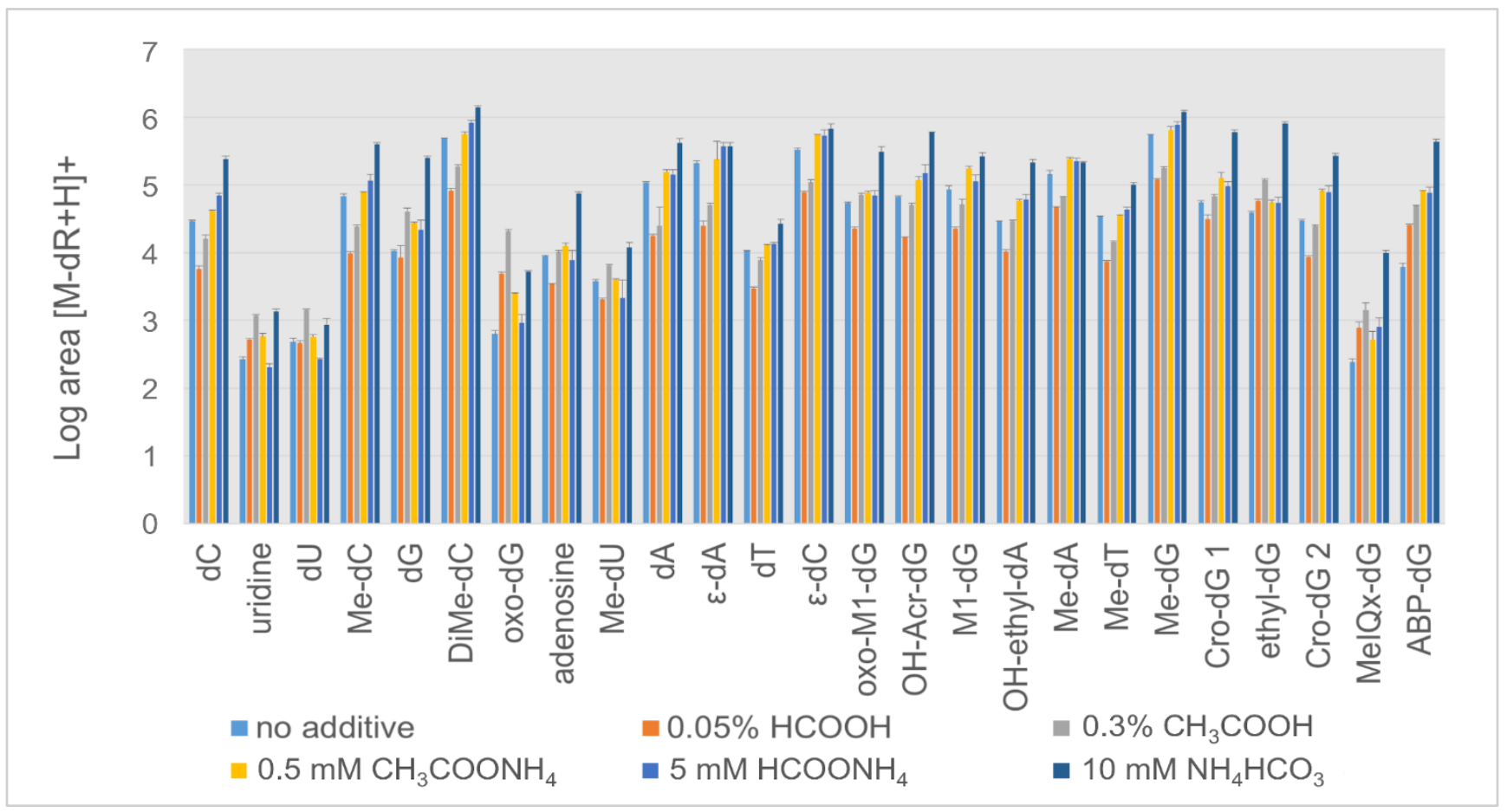

Figure1: $\mathrm{Log}$ of the area of the $[\mathrm{M}-\mathrm{dR}+\mathrm{H}]+$ ion of the DNA adduct standards, analyzed using the following additives in the mobile phases: no additive, $0.05 \% \mathrm{HCOOH}, 0.3 \% \mathrm{CH} 3 \mathrm{COOH}, 5 \mathrm{mM} \mathrm{CH} 3 \mathrm{COONH} 4,5 \mathrm{mM} \mathrm{HCOONH} 4$, and 10mM NH4HCO3.

$[\mathrm{M}-\mathrm{dR}+\mathrm{H}]^{+}$was almost constant, and was therefore not the discriminant parameter for the mobile phase evaluation. Increasing $\mathrm{HCOOH}$ concentration lowered the intensity of $[\mathrm{M}+\mathrm{H}]^{+}$and $[\mathrm{M}-\mathrm{dR}+\mathrm{H}]$ and raised the intensity of $[\mathrm{M}+\mathrm{Na}]^{+}$for most compounds, so a concentration $0.05 \% \mathrm{HCOOH}$ was chosen for further comparisons (Figure S4.1). There was no particular trend with the concentration of $\mathrm{CH}_{3} \mathrm{COOH}$ but the highest signal was obtained at $0.3 \%$ (Figure S4.2), which also showed less $[\mathrm{M}+\mathrm{Na}]^{+},[\mathrm{M}+\mathrm{K}]^{+},[2 \mathrm{M}+\mathrm{H}]^{+}$ and $[2 \mathrm{M}+\mathrm{Na}]^{+}$, and therefore was chosen as the best condition. The area of $[\mathrm{M}+\mathrm{H}]^{+}$was increased by decreasing the concentration of $\mathrm{CH}_{3} \mathrm{COONH}_{4}$ for all compounds other than thymine, Me-dC, and M1-dG (Figure S4.3). An increase in the formation of $\left[\mathrm{M}+\mathrm{C}_{2} \mathrm{H}_{6} \mathrm{~N}_{2}+\mathrm{H}\right]^{+}$, possibly due to a contaminant such as acetamidine, ${ }^{26}$ was detected for some of the

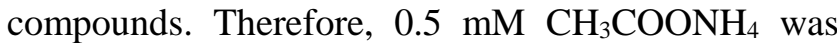
chosen as the best condition. In the case of $\mathrm{HCOONH}_{4}$ most of the compounds reached a maximum signal at $5 \mathrm{mM} \mathrm{HCOONH} 4$ (Figure S4.4), which was chosen as the best condition. Finally, $\mathrm{NH}_{4} \mathrm{HCO}_{3}$ showed very heterogeneous behavior across all compounds (Figure S4.5). Therefore, $10 \mathrm{mM} \mathrm{NH}_{4} \mathrm{HCO}_{3}$ was chosen, giving the highest average signal among the DNA adducts. In conclusion, $0.05 \% \mathrm{HCOOH}, 0.3 \% \mathrm{CH}_{3} \mathrm{COOH}, 5 \mathrm{mM}$
$\mathrm{CH}_{3} \mathrm{COONH}_{4}, \quad 5 \mathrm{mM} \quad \mathrm{HCOONH}_{4}$, and $10 \mathrm{mM}$ $\mathrm{NH}_{4} \mathrm{HCO}_{3}$ were chosen for further comparison.

As shown in Figure 1, the best mobile phase for the majority of the DNA adducts was $10 \mathrm{mM} \mathrm{NH}_{4} \mathrm{HCO}_{3}$, showing an increase of up to 2 orders of magnitude compared to the other conditions. This was due to the ability of $\mathrm{NH}_{4} \mathrm{HCO}_{3}$ to suppress the formation of $[\mathrm{M}+\mathrm{Na}]^{+}$and $[\mathrm{M}+\mathrm{K}]^{+}$to a much greater extent than the other additives. Both the number of DNA adducts forming $\mathrm{Na}^{+}$and $\mathrm{K}^{+}$adducts and the area of the $\mathrm{Na}^{+}$and $\mathrm{K}^{+}$ions were lower than in the other conditions (Figures S4.1-S4.5). Only uridine, dU, and oxo-dG, showed a better response when $\mathrm{CH}_{3} \mathrm{COOH}$ was used. Under almost all conditions, uridine, $\mathrm{dU}$ and oxo-dG, together with Me-dU, dT, and Me-dT, showed only $[\mathrm{M}+\mathrm{Na}]^{+}$ and very little $[\mathrm{M}+\mathrm{H}]^{+}$, due to their acidic properties..$^{27,28}$ Nucleosides that can exist in one or more tautomeric hydroxy forms, such as thymine (1 form), uracil ( 2 forms), and oxo-dG ( 2 forms), easily lose their proton in an alkaline medium such as $10 \mathrm{mM} \mathrm{NH}_{4} \mathrm{HCO}_{3}$ ( $\mathrm{pH}$ 9.35). Uracil- and thymine-derived adducts also showed a better response in negative polarity (data not shown), suggesting the need for separate acquisition and optimization in future work. The chromatographic parameters did not affect performance for any of the mobile phases. All tested conditions gave optimal 
retention, peak shape, resolution, and reproducibility. (Figures S4.6, S4.7, S4.8, Table S4.1).

In order to further reduce the formation of $[\mathrm{M}+\mathrm{Na}]^{+}$or $[\mathrm{M}+\mathrm{K}]^{+}$, a final comparison was performed using different mobile phase bottles, i.e. common glass bottles vs low density polyethylene LDPE bottles (Waters). A slight reduction of $[\mathrm{M}+\mathrm{Na}]^{+}$and a clear reduction of $[\mathrm{M}+\mathrm{K}]^{+}$was found for most of the adducts when using the LDPE bottles. However, this did not improve the sensitivity of the method as the intensity of the $[\mathrm{M}+\mathrm{H}]^{+}$remained constant (Figure S4.9).

\section{Optimization of Mass Spectrometric Conditions}

Once the best chromatographic condition was chosen, a comprehensive mass spectrometric optimization was carried out to increase the intensity of the signal and reduce the loss of $-\mathrm{dR}$ from the DNA adducts. The parameters investigated included ESI related parameters (capillary and sampling cone voltage, source temperature, desolvation gas flow and temperature), collision energies (the minimum required to ensure the transmission of the ions in qTOF), acquisition mode related parameters (mass range, the use of automatic or manual profile mode, i.e. automatically or manually optimizing the quadrupole parameters), and detector parameters (detector voltage). The results of the mass spectrometric optimization are shown in terms of both the intensity of the most abundant ion for each DNA adduct, and the ratio between that ion and the DNA adduct after the loss of $-\mathrm{dR}$ (Figure $S$ 5.1). Increasing the capillary and sampling cone voltages resulted in a remarkable decrease of the signal and increase of the loss of $-\mathrm{dR}$. A minimum value was therefore chosen for the capillary voltage, and a value of $20 \mathrm{eV}$ was chosen for the cone voltage. An increase in the signal was seen when the desolvation gas temperature was increased. The mass range did not affect the signal greatly, and automatic profile was better than manual. Changing the source temperature, the desolvation gas flow, and the collision energy did not produce any particular improvement. The signal was strongly affected by the detector voltage, which was optimal at $3000 \mathrm{~V}$. This increased the signal by up to 10 times. However, using detector voltage higher than the limit recommended by the vendor specialists could damage the detector. In conclusion, minimizing capillary and sampling cone voltages, and raising detector voltage caused the most remarkable increase in the signal (Figure 2). A partial reduction of $-\mathrm{dR}$ loss was achieved by reducing capillary and sampling cone voltages.

\section{Reduction of adsorption processes}

Many other factors, besides the ionization efficiency in the source, can affect the sensitivity of the method. For compounds present in traces such as DNA adducts, adsorption processes can occur on several surfaces, such as the injection vials, the metal surfaces of the

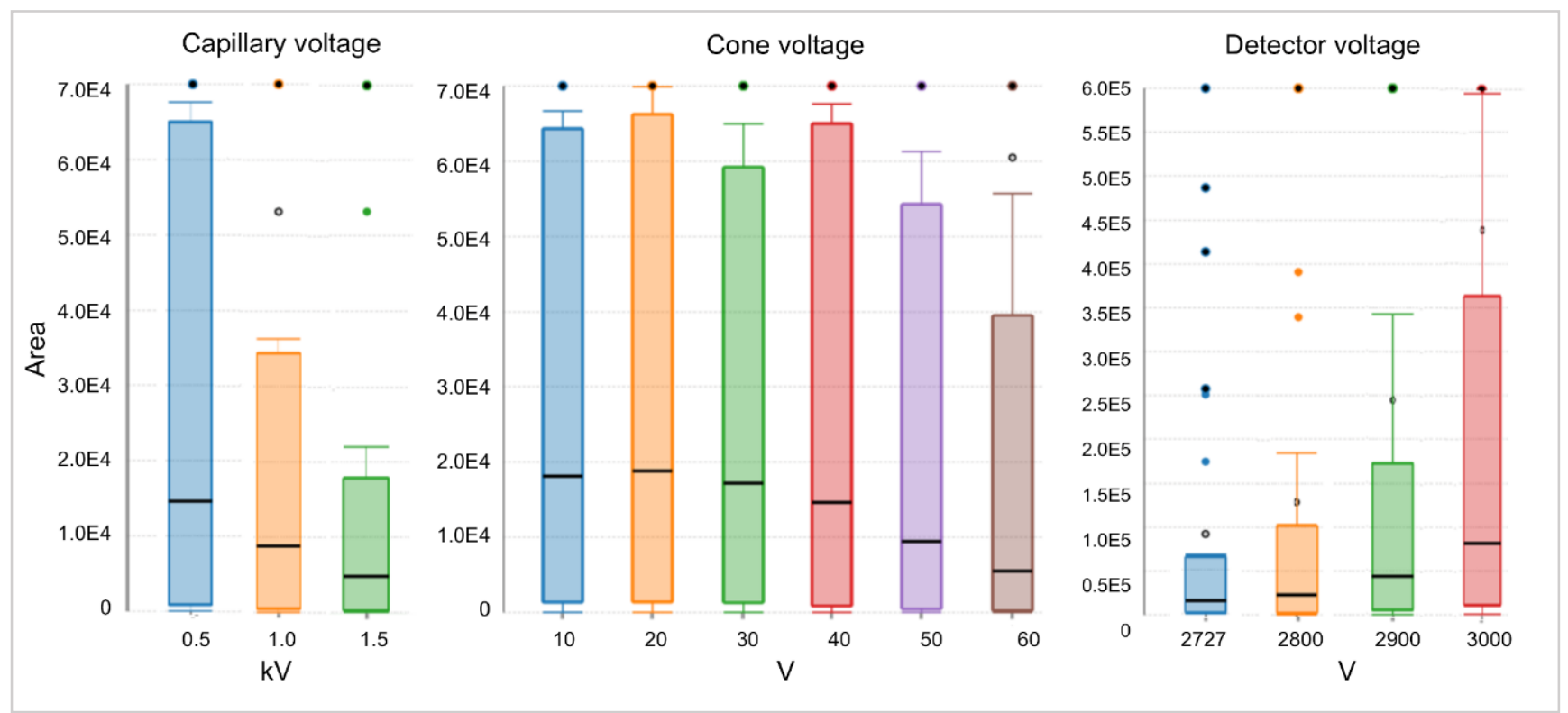

Figure 2: Box-and-Whisker plots showing the ditribution of the peak area of the DNA adduct standards at different values of capillary, sampling cone, and detector voltage (Graph created in www.goodcalculators.com). 
chromatographic system and the column. Several precautions were taken for controlling adsorption processes in the optimization process. However, the results often showed a discrete change in the intensity over continuous injection series. To explore this issue, 5 different injection vials and 2 different columns were evaluated. Four different low-adsorptive injection vials were compared with a standard glass vial (LC-MS certified). The standard glass vial displayed the highest instability, with a clear signal decrease after the first hour for several of the DNA adducts (Figure S6.1). The RSA-Pro vial gave the highest signal for most of the compounds, but the signal tended to increase after the first hour, and then decrease after 4 hours of injections. The other three vials showed a quite stable signal over time, generally up to 16 hours. However, the Quan Recovery vial gave a much lower signal for M1-dG and Me-dG compared to the other vials, whereas the TruView vial showed a lower signal for ABP-dG and no signal for MeIQx-dG, suggesting that the TruView vial is not suitable for the analysis of bulky DNA adducts. In conclusion, the vial showing an acceptable behavior in terms of both intensity and stability for DNA-adduct analysis is the Low Adsorption vial.

The chromatographic column chosen for developing this method, HSST3, has recently been marketed as Premier HSST3, where a hybrid organic-inorganic surface, based on an ethylene-bridged siloxane chemistry has been applied on the metal surfaces for reducing the adsorption of nucleotides. ${ }^{29}$ In this work, the HSST3 and Premier HSST3 were tested for separating DNA adduct nucleosides, by using mobile phases containing either $0.5 \mathrm{mM} \mathrm{CH}_{3} \mathrm{COONH}_{4}(\mathrm{pH}$ 6.27) or $10 \mathrm{mM} \mathrm{NH}_{4} \mathrm{HCO}_{3}(\mathrm{pH} 9.35)$. The $10 \mathrm{mM}$ $\mathrm{NH}_{4} \mathrm{HCO}_{3}$ was tested in duplicate on another Premier HSST3. With $\mathrm{CH}_{3} \mathrm{COONH}_{4}$ the most acidic DNA adducts, such as uridine, Me-dU, and dU, showed a remarkable improvement in the Premier HSST3. This can be explained by a reduction of the adsorption of acidic negatively charged analytes on the metal oxide layer of the 'native' HSST3, which is positively charged at $\mathrm{pH}<7 .{ }^{27}$ (Figure S6.2). With both $\mathrm{CH}_{3} \mathrm{COONH}_{4}$ and $\mathrm{NH}_{4} \mathrm{HCO}_{3}$, a remarkable increase of the signal was showed for MeIQx-dG when using the new premierHSST3, and for oxo-dG, ABP-dG only in one of the two duplicates. However, most of the other DNA adducts showed a better performance of the HSST3.
Probably an interaction with the hybrid surface occurs for most DNA adducts besides the bulky ones. However, further studies are needed to understand the nature of the interactions. In conclusion, since the HSST3 showed generally better performance compared to the Premier HSST3, the former was chosen for further analysis. However, the use of the Premier HSST3 with acidic mobile phases is suggested for the analysis of acidic DNA adducts.

\section{Acquisition mode and identification approach}

Several approaches have been employed in the past for screening of DNA adducts, often by monitoring the loss of - $\mathrm{dR}$ and the unmodified nucleobase fragment ions. Early DNA adductomics primarily utilized triple quadrupole instrumentation to perform neutral loss screening, ${ }^{30}$ whereas more recent studies have taken advantage of HRMS, ${ }^{4}$ which allows performing different types of acquisition modes such as DDAtriggered neutral loss, ${ }^{9}$ wide selected ion monitoring tandem mass spectrometry (Wide-SIM/MS2) ${ }^{10}$ and data independent acquisition (DIA). ${ }^{6}$ Whereas DDA selects specific precursor ions for fragmentation resulting in clean MS2 spectra, DIA (or $\mathrm{MS}^{\mathrm{E}}$ ) fragments the entire range of ions, requiring elaborate

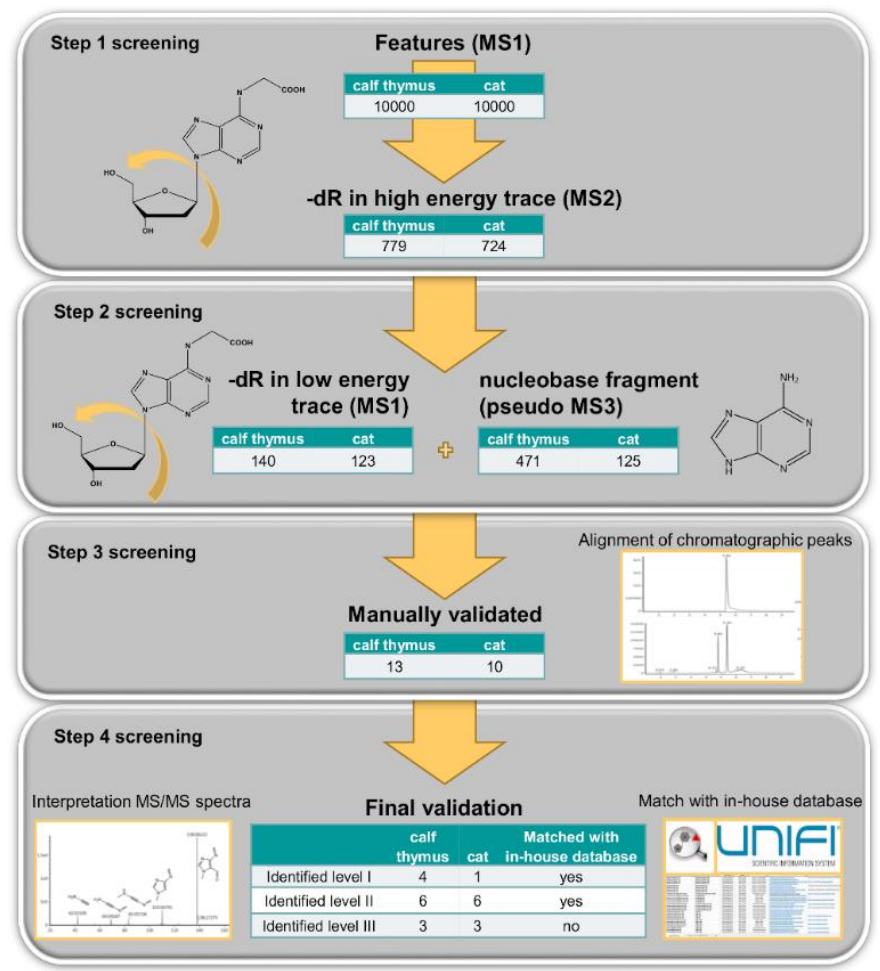

Figure 3: Summary of the selected features and tentatively identified DNA adducts in DNA from calf thymus and cat colon. 
data analysis software for the investigation of the spectra ${ }^{6}$ and a supplementary MS2 targeted acquisition for confirming the identity of the compound. However, the selective approach of DDA carries the risk of losing the fragmentation of the least abundant compounds. ${ }^{4}$ This is important for DNA adducts, which are normally present as traces in complex biological matrices rich in highly abundant contaminants. To allow detection and fragmentation of a high number of features, $\mathrm{MS}^{\mathrm{E}}$ acquisition at 6 and 20$50 \mathrm{eV}$ was therefore chosen for the low and high energy traces, respectively. The first step of screening (Figure 3) consisted in monitoring the loss of $-\mathrm{dR}$ across all the features in the high energy trace (MS2) to find potential DNA adducts. Since all the DNA adduct standards showed the in-source fragment [M$\mathrm{dR}+\mathrm{H}]^{+}$in the low energy trace (MS1), a pseudo $\mathrm{MS}^{3}$ fragmentation could be performed, where the modified nucleobase could be further fragmented in the high energy collision trace (MS2) leading to the loss of the modification. Therefore, a second step of screening could be introduced, consisting in monitoring the loss of $-\mathrm{dR}$ in the low energy trace (MS1) and the presence of the fragment ions belonging to the unmodified nucleobases in the high energy trace (pseudo-MS3). Not all the DNA adducts showed abundant in-source fragmentation, so features showing either loss of $-\mathrm{dR}$ in low energy trace or typical nucleobases fragment ions were selected for further validation. The third level of screening consisted in a manual inspection of the chromatographic peak shapes of the $[\mathrm{M}+\mathrm{H}]^{+}$, the $[\mathrm{M}-\mathrm{dR}+\mathrm{H}]^{+}$in $\mathrm{MS} 1$ and MS2, and the nucleobase fragments in MS2. After manual validation, the number of matches remarkably decreased since the loss of - $\mathrm{dR}$ across the low and high energy trace can belong to different molecules, due the unselective fragmentation of the $\mathrm{MS}^{\mathrm{E}}$ acquisition. The manual validation could be avoided by implementing the software with a peak picking function in both traces before the search for $-\mathrm{dR}$ loss. Finally, the selected masses were matched with our in-house DNA-adducts database and further fragmented through targeted MS2 (fourth step of screening). A summary of the selected features and tentatively identified DNA adducts is provided in Figure 3.

\section{Identification in DNA from calf thymus and cat colon tissue}

The optimized untargeted method was applied for the identification of DNA adducts from calf thymus and from cat colon, leading to the tentative identification of 13 possible DNA adducts as shown in Table S7.1 and 7.2 .

C8-oxo-dG, dU and 5-Me-dC were compared with commercial reference standards and identified at level I in commercial calf thymus DNA and freshly isolated cat colon DNA. C8-oxo-dG is one of the most studied DNA adducts and it derives from the oxidative damage to DNA caused by reactive oxygen species (ROS), which occurs endogenously as part of normal metabolism. ${ }^{31} \mathrm{dU}$ is a result of deamination of cytosine to uracil, which occurs spontaneously as a result of several mechanisms such as simple hydrolysis, attack by nitric oxide-derived species or ROS during inflammation, and by the activity of deaminase enzymes. $^{32,33} 5-\mathrm{Me}-\mathrm{dC}$ is a result of epigenetic modification processes. ${ }^{34}$ High abundance of these adducts is therefore expected.

Two isomers of carboxy-Me-dG and two isomers of carboxyethyl-dG were fragmented through targeted MS2 and tentatively identified at level II in DNA from calf thymus and from cat colon. The two isomers are probably due to different positions of the carboxymethyl and carboxyethyl groups on the dG. However, the identities are difficult to confirm, as peaks partially overlap, showing the same fragmentation pattern. Carboxy-Me-dG and carboxyethyl-dG have the same accurate mass as the adducts from common glycotoxins, glyoxal-dG and Me-glyoxal-dG. However, in both cases and in both isomers, the loss of $\mathrm{CO}_{2}$ in the fragmentation pattern strongly suggests the identities reported here. Glyoxal$\mathrm{dG}$ has been found to be unstable and is partially transformed to carboxy-Me-dG. ${ }^{35}$ The compounds may therefore derive mainly from rearrangements of adducts formed by known glycotoxins. In previous works, O6-carboxy-Me-dG has been found in colon tissues $^{8}$ and exfoliated colonocytes. ${ }^{36}$ Me-glyoxal is a common byproduct of the ubiquitous glycolysis pathway and reacts with $\mathrm{dG}$ to form $\mathrm{N}^{2}$-carboxyethyldG. ${ }^{37}$

Deoxyxanthosine and deoxyinosine were also identified at level II in DNA from both calf thymus and 
cat colon. These two adducts are a result of $\mathrm{dG}$ and $\mathrm{dA}$ deamination in DNA, respectively, as in the case of dU. ${ }^{32,33}$

Finally, four unknown adducts were detected in DNA from calf thymus, two of them modifications of with cytosine, one of hypoxanthine, one is uncertain. However, the fragmentation pattern was not sufficiently informative and they have been assigned at level III. The detection of the four unknown DNA adducts show the potential of the method and the identification workflow for performing untargeted DNA adductomics.

In the only reported DNA adductomics study on colon tissues, 17 DNA adducts were tentatively identified, including O6-Carboxy-Me-dG and other adducts not found in the current work. ${ }^{8}$ However, nucleobases obtained after acidic hydrolysis were analysed rather than nucleosides. In lung tissue ${ }^{9}$ or carcinogen-treated HT-29 adenocarcinoma cells, ${ }^{22}$ a range of 30-50 DNA adducts were tentatively identified. Here different sample preparation and instrumental methods, including nano-ESI-HPLC and fractionation, were used for the analysis. Finally, in a work from Cooke et al., ${ }^{30} 16$ DNA adduct nucleosides and nucleobases have been detected in urine by using an LC-QqQ. The range of DNA adducts identified in the current work is in agreement with the existing literature. Moreover, only few untargeted DNA adductomics studies have shown the possibility of identifying new DNA adducts, ${ }^{10-13}$ indicating the current $\mathrm{MS}^{\mathrm{E}}$ based method and identification workflow to be promising for performing untargeted DNA adductomics. Improved sensitivity of the analytical method by using nanoHPLC and by optimizing the sample preparation will further enhance this technology for translation into cancer research.

\section{- CONCLUSIONS}

In this work, a sensitive UHPLC-HRMS untargeted method was developed for the detection and identification of DNA adducts. The optimization of the chromatographic conditions showed the mobile phases containing $10 \mathrm{mM} \mathrm{NH}_{4} \mathrm{HCO}_{3}$ to be the best condition in terms of signal enhancement of a mix of DNA adducts used as reference standards. The capillary voltage, the sampling cone voltage, and the detector voltage highly affected the response of the DNA adducts. Also, a low adsorption vial was selected for the highest stability of the signal over time. These optimised analytical conditions were chosen for the analysis of DNA from calf thymus and cat colon using $\mathrm{MS}^{\mathrm{E}}$ acquisition, where the DNA adducts were screened by monitoring the loss of -dR both in-source and in the fragmentation spectra, allowing further fragmentation of the ions belonging to the unmodified nucleobases. The putative DNA adducts were matched with an in-house database. The method led to the identification of 13 DNA adducts in DNA from calf thymus and cat colon. Four of these have never been reported before, showing good promise for the application of this untargeted method in future human studies. The current method should be applied to analysis of human colon DNA to improve understanding of the adducts potentially responsible of causing genetic damage to the tissue.

\section{Acknowledgments}

This work, and the fellowship of Giorgia La Barbera, were funded by the European Commision for the H2020 Marie Curie Slodowska Individual Fellowships (grant $n^{\circ}$ 843892). Professor Lars Ove Dragsted was supported by a Semper Ardens grant (CF15-0574) from the Carlsberg Foundation. Professor Charlotte Reinhard Bjørnvad, Department of Veterinary Clinical Science, University of Copenhagen, provided the cat colon sample.

\section{References}

(1) Balbo, S.; Turesky, R. J.; Villalta, P. W. Chem. Res. Toxicol. 2014, 27 (3), 356-366.

(2) Loeb, L. A.; Harris, C. C. Cancer Res. 2008, 68 (17), 6863.

(3) Tretyakova, N.; Villalta, P. W.; Kotapati, S. Chem. Rev. 2013, 113 (4), 2395-2436.

(4) Villalta, P.; Balbo, S. Int. J. Mol. Sci. 2017, 18 (9), 1870.

(5) Murray, K. J.; Carlson, E. S.; Stornetta, A.; Balskus, E. P.; Villalta, P. W.; Balbo, S. Anal. Chem. 2021, 93 (14), 5754-5762.

(6) Sousa, P. F. M.; Martella, G.; Åberg, K. M.; Esfahani, B.; Motwani, H. V. Toxics 2021, Vol. 9, Page 78 2021, 9 (4), 78.

(7) Hemeryck, L. Y.; Vanhaecke, L. Nutr. Rev. 2016, 74 (8), 475-489.

(8) Hemeryck, L. Y.; Decloedt, A. I.; Vanden Bussche, J.; Geboes, K. P.; Vanhaecke, L. Anal. Chim. Acta 2015, 892, 123-131. 
(9) Carrà, A.; Guidolin, V.; Dator, R. P.; Upadhyaya, P.; Kassie, F.; Villalta, P. W.; Balbo, S. Front. Chem. 2019, 7, 658.

(10) Guo, J.; Villalta, P. W.; Turesky, R. J. Anal. Chem. 2017, 89 (21), 11728-11736.

(11) Wilson, M. R.; Jiang, Y.; Villalta, P. W.; Stornetta, A.; Boudreau, P. D.; Carrá, A.; Brennan, C. A.; Chun, E.; Ngo, L.; Samson, L. D.; Engelward, B. P.; Garrett, W. S.; Balbo, S.; Balskus, E. P. Science (80-. ). 2019, 363 (6428).

(12) Guidolin, V.; Carlson, E. S.; Carrà, A.; Villalta, P. W.; Maertens, L. A.; Hecht, S. S.; Balbo, S. Biomolecules 2021, 11 (3), 1-20.

(13) Guo, J.; Villalta, P. W.; Weight, C. J.; Bonala, R.; Johnson, F.; Rosenquist, T. A.; Turesky, R. J. Chem. Res. Toxicol. 2018, 31 (12), 1382-1397.

(14) Tudek, B.; Speina, E. Mutat. Res. Mol. Mech. Mutagen. 2012, 736 (1-2), 82-92.

(15) Murphy, N.; Moreno, V.; Hughes, D. J.; Vodicka, L.; Vodicka, P.; Aglago, E. K.; Gunter, M. J.; Jenab, M. Mol. Aspects Med. 2019, 69, 29.

(16) Chambers, M. C.; MacLean, B.; Burke, R.; Amodei, D.; Ruderman, D. L.; Neumann, S.; Gatto, L.; Fischer, B.; Pratt, B.; Egertson, J.; Hoff, K.; Kessner, D.; Tasman, N.; Shulman, N.; Frewen, B.; Baker, T. A.; Brusniak, M. Y.; Paulse, C.; Creasy, D.; Flashner, L.; Kani, K.; Moulding, C.; Seymour, S. L.; Nuwaysir, L. M.; Lefebvre, B.; Kuhlmann, F.; Roark, J.; Rainer, P.; Detlev, S.; Hemenway, T.; Huhmer, A.; Langridge, J.; Connolly, B.; Chadick, T.; Holly, K.; Eckels, J.; Deutsch, E. W.; Moritz, R. L.; Katz, J. E.; Agus, D. B.; MacCoss, M.; Tabb, D. L.; Mallick, P. Nature Biotechnology. 2012, 30 (10) 918-920.

(17) Pluskal, T.; Castillo, S.; Villar-Briones, A.; Orešič, M. BMC Bioinformatics 2010, 11.

(18) La Barbera, G.; Antonelli, M.; Cavaliere, C.; Cruciani, G.; Goracci, L.; Montone, C. M.; Piovesana, S.; Laganà, A.; Capriotti, A. L. Anal. Chem. 2018, 90 (20), 12230-12238.

(19) Schymanski, E. L.; Jeon, J.; Gulde, R.; Fenner, K.; Ruff, M.; Singer, H. P.; Hollender, J. Environ. Sci. Technol. 2014, 48 (4), 2097-2098.

(20) Villalta, P. W.; Hochalter, J. B.; Hecht, S. S. Ultrasensitive High-Resolution Mass. Anal. Chem. 2017, 89 (23), 12735-12742.

(21) Balbo, S.; Hecht, S. S.; Upadhyaya, P.; Villalta, P. W. Anal. Chem. 2014, 86 (3), 1744-1752.

(22) Stornetta, A.; Villalta, P. W.; Hecht, S. S.; Sturla, S. J.; Balbo, S. Anal. Chem. 2015, 87 (23), 11706-11713.

(23) Hemeryck, L. Y.; Rombouts, C.; De Paepe, E.;
Vanhaecke, L. Food Chem. Toxicol. 2018, 115, 73-87.

(24) Yin, R.; Liu, S.; Zhao, C.; Lu, M.; Tang, M.-S.; Wang, H. 2013, 85 (6), 3190-3197

(25) Murakami, H.; Horiba, R.; Iwata, T.; Miki, Y.; Uno, B.; Sakai, T.; Kaneko, K.; Ishihama, Y.; Teshima, N.; Esaka, Y. Talanta 2018, 177, 1217.

(26) Abián, J.; Sáchez-Baeza, F.; Gelpí, E.; Barceló, D. J. Am. Soc. Mass Spectrom. 1994, 5 (3), 186193.

(27) Liguori, A.; Napoli, A.; Sindona, G. J. Mass Spectrom 2000, 35, 139-144.

(28) von Sonntag, C. In Free-Radical-Induced DNA Damage and Its Repair; Springer, Berlin, Heidelberg, 2006, 211-334.

(29) DeLano, M.; Walter, T. H.; Lauber, M. A.; Gilar, M.; Jung, M. C.; Nguyen, J. M.; Boissel, C.; Patel, A. V.; Bates-Harrison, A.; Wyndham,K. D. Anal. Chem. 2021, 93 (14), 5773-5781.

(30) Cooke, M. S.; Hu, C. W.; Chang, Y. J.; Chao, M. R. Environment International. Environ Int, 2018, 121 (2), 1033-1038.

(31) Dizdaroglu, M.; Jaruga, P. 2012, 46 (4), 382419.

(32) Pang, B.; McFaline, J. L.; Burgis, N. E.; Dong, M.; Taghizadeh, K.; Sullivan, M. R.; Elmquist, C. E.; Cunningham, R. P.; Dedon, P. C. Proc. Natl. Acad. Sci. 2012, 109 (7), 2319-2324.

(33) Kow, Y. W. Repair of Deaminated Bases in DNA. Free Radic. Biol. Med. 2002, 33 (7), 886893.

(34) Kumar, S.; Chinnusamy, V.; Mohapatra, T. Front. Genet. 2018, 9, 640.

(35) Wang, H.; Cao, H.; Wang, Y. Chem. Res. Toxicol. 2010, 23 (1), 74.

(36) Lewin, M. H.; Bailey, N.; Bandaletova, T.; Bowman, R.; Cross, A. J.; Pollock, J.; Shuker, D. E. G.; Bingham, S. A. Cancer Res. 2006, 66 (3), 1859-1865.

(37) Frischmann, M.; Bidmon, C.; Angerer, J.; Pischetsrieder, M. Chem. Res. Toxicol. 2005, 18 (10), 1586-1592. 